\title{
Identifying codes of lexicographic product of graphs
}

\author{
Min Feng Min Xu Kaishun Wang* \\ Sch. Math. Sci. \& Lab. Math. Com. Sys. \\ Beijing Normal University, Beijing 100875, China \\ fengmin@mail.bnu.edu.cn $\quad$ xum, wangks $\} @ b n u . e d u . c n$ \\ Submitted: Jun 26, 2011; Accepted: Dec 13, 2012; Published: Dec 31, 2012 \\ Mathematics Subject Classifications: 94A29, 05C90
}

\begin{abstract}
Let $G$ be a connected graph and $H$ be an arbitrary graph. In this paper, we study the identifying codes of the lexicographic product $G[H]$ of $G$ and $H$. We first introduce two parameters of $H$, which are closely related to identifying codes of $H$. Then we provide the sufficient and necessary condition for $G[H]$ to be identifiable. Finally, if $G[H]$ is identifiable, we determine the minimum cardinality of identifying codes of $G[H]$ in terms of the order of $G$ and these two parameters of $H$.
\end{abstract}

Key words: identifying code; lexicographic product.

\section{Introduction}

In this paper, we only consider finite undirected simple graphs with at least two vertices. For a given graph $G$, we often write $V(G)$ for the vertex set of $G$ and $E(G)$ for the edge set of $G$. For any two vertices $u$ and $v$ of $G$, let $d_{G}(u, v)$ denote the distance between $u$ and $v$ in $G$. Given a vertex $v \in V(G)$, define $B_{G}(v)=\left\{u \mid u \in V(G), d_{G}(u, v) \leqslant 1\right\}$. A code $C$ is a nonempty set of vertices. We say that a code $C$ covers $v$ if $B_{G}(v) \cap C \neq \emptyset$; we say that $C$ separates two distinct vertices $x$ and $y$ if $B_{G}(x) \cap C \neq B_{G}(y) \cap C$. An identifying code of $G$ is a code which covers all the vertices of $G$ and separates any pair of distinct vertices of $G$. If $G$ admits at least one identifying code, we say $G$ is identifiable and denote the minimum cardinality of all identifying codes of $G$ by $I(G)$.

The concept of identifying codes was introduced by Karpovsky et al. [12] to model a fault-detection problem in multiprocessor systems. It was noted in $[4,5]$ that determining the identifying code with the minimum cardinality in a graph is an NP-complete problem.

${ }^{*}$ Corresponding author. 
Many researchers have focused on the study of identifying codes in some restricted classes of graphs, for example, paths [2], cycles [2, 7, 16], hypercubes $[3,11,13,14]$ and infinite grids $[1,6,10]$.

Gravier et al. [8] investigated the identifying codes of Cartesian product of two cliques. Rall and Wash [15] studied the identifying codes of the direct product of two cliques. In this paper, we study the identifying codes of the lexicographic product $G[H]$ of a connected graph $G$ and an arbitrary graph $H$. In Section 2, we introduce two parameters of a graph which are closely related to identifying codes, and compute these two parameters of the path $P_{n}$ and the cycle $C_{n}$, respectively. In Section 3, we first provide the sufficient and necessary condition for $G[H]$ to be identifiable, then determine $I(G[H])$ in terms of the order of $G$ and the two parameters of $H$ when $G[H]$ is identifiable. In particular, the values of $I\left(G\left[P_{n}\right]\right)$ and $I\left(G\left[C_{n}\right]\right)$ are determined.

\section{Two parameters}

For a graph $H$, let $C^{\prime} \subseteq V(H)$ be a code which separates any pair of distinct vertices of $H$, we use $I^{\prime}(H)$ to denote the minimum cardinality of all possible $C^{\prime}$. This code was studied in [3]. Let $C^{\prime \prime} \subseteq V(H)$ be a code which separates any pair of distinct vertices of $H$ and satisfies $C^{\prime \prime} \nsubseteq B_{H}(v)$ for every $v \in V(H)$, we use $I^{\prime \prime}(H)$ to denote the minimum cardinality of all possible $C^{\prime \prime}$.

The two parameters $I^{\prime}(H)$ and $I^{\prime \prime}(H)$ are used to compute the minimum cardinality of identifying codes of $G[H]$ of graphs $G$ and $H$ (see Theorem 3.4). In this section we shall compute the two parameters for paths and cycles, respectively.

Given an integer $n \geqslant 3$, suppose

$$
\begin{gathered}
V\left(P_{n}\right)=\{0,1, \ldots, n-1\}, E\left(P_{n}\right)=\{i j \mid j=i+1, i=0, \ldots, n-2\} \\
V\left(C_{n}\right)=\mathbb{Z}_{n}=\{0,1, \ldots, n-1\}, E\left(C_{n}\right)=\left\{i j \mid j=i+1, i \in \mathbb{Z}_{n}\right\}
\end{gathered}
$$

Example 2.1 $I^{\prime}\left(P_{3}\right)=2$ and $I^{\prime \prime}\left(P_{3}\right)$ is not well defined; $I^{\prime}\left(P_{4}\right)=3$ and $I^{\prime \prime}\left(P_{4}\right)=4$; $I^{\prime}\left(P_{5}\right)=I^{\prime \prime}\left(P_{5}\right)=3 ; I^{\prime}\left(P_{6}\right)=3$ and $I^{\prime \prime}\left(P_{6}\right)=4$.

For $P_{4},\{0,1,2\}$ is an identifying code, but $\{0,1,2\} \subseteq B_{P_{4}}(1)$ and $\{0,1,3\}$ can not separate 0 and 1 . For $P_{5},\{0,2,4\}$ separates any pair of distinct vertices. For $P_{6},\{1,2,3\}$ separates any pair of distinct vertices, but $\{1,2,3\} \subseteq B_{P_{6}}(2)$.

Example 2.2 $I^{\prime}\left(C_{4}\right)=3$ and $I^{\prime \prime}\left(C_{4}\right)=4 ; I^{\prime}\left(C_{5}\right)=3$ and $I^{\prime \prime}\left(C_{5}\right)=4 ; I^{\prime}\left(C_{6}\right)=I^{\prime \prime}\left(C_{6}\right)=$ $3 ; I^{\prime}\left(C_{7}\right)=I^{\prime \prime}\left(C_{7}\right)=4 ; I^{\prime}\left(C_{9}\right)=I^{\prime \prime}\left(C_{9}\right)=6 ; I^{\prime}\left(C_{11}\right)=I^{\prime \prime}\left(C_{11}\right)=6$.

For $C_{4},\{0,1,2\}$ is an identifying code, but $\{0,1,2\} \subseteq B_{C_{4}}(1)$. For $C_{5},\{0,1,2\}$ is an identifying code, but $\{0,1,2\} \subseteq B_{C_{5}}(1)$ and $\{0,1,3\}$ can not separate 0 and 1 . For $C_{6}$, both $\{3,4,5\}$ and $\{0,2,4\}$ separate any pair of distinct vertices. For $C_{7},\{3,4,5,6\}$ separates any pair of distinct vertices. For $C_{9}$, both $\{3,4,5,6,7,8\}$ and $\{0,2,4,6,7,8\}$ separate any pair of distinct vertices. For $C_{11},\{3,4,5,8,9,10\}$ separates any pair of distinct vertices. 

$[2,7]$.

The minimum cardinality of identifying codes of a path or a cycle was computed in

Proposition 2.1 ([2, 7]) (i) For $n \geqslant 3, I\left(P_{n}\right)=\left\lfloor\frac{n}{2}\right\rfloor+1$;

(ii) For $n \geqslant 6, I\left(C_{n}\right)= \begin{cases}\frac{n}{2}, & n \text { is even, } \\ \frac{n+3}{2}, & n \text { is odd. }\end{cases}$

In order to compute the two parameters for paths and cycle, we need the following useful lemma.

Lemma 2.2 Let $H$ be an identifiable graph.

(i) $I(H)-1 \leqslant I^{\prime}(H) \leqslant I(H)$;

(ii) If $\Delta(H) \leqslant|V(H)|-2$, then $I(H)-1 \leqslant I^{\prime}(H) \leqslant I^{\prime \prime}(H) \leqslant I(H)+1$, where $\Delta(H)$ is the maximum degree of $H$.

Proof. Let $C^{\prime}$ be a code which separates any pair of distinct vertices of $H$.

(i) Since there exists at most one vertex $v$ not covered by $C^{\prime}, C^{\prime} \cup\{v\}$ is an identifying code of $H$. Then $I(H) \leqslant I^{\prime}(H)+1$, as desired.

(ii) Note that there exists at most one vertex $v$ such that $C^{\prime} \subseteq B_{H}(v)$. Since $\Delta(H) \leqslant$ $|V(H)|-2$, there exists $v_{0} \in V(H) \backslash B_{H}(v)$ such that $C^{\prime \prime}=C^{\prime} \cup\left\{v_{0}\right\}$ is a code which separates any pair of distinct vertices of $H$ and satisfies $C^{\prime \prime} \nsubseteq B_{H}(w)$ for every $w \in V(H)$. It follows that $I^{\prime}(H) \leqslant I^{\prime \prime}(H) \leqslant I^{\prime}(H)+1$. By (i), (ii) holds.

Proposition 2.3 For $n \geqslant 7, I^{\prime}\left(P_{n}\right)=I^{\prime \prime}\left(P_{n}\right)=\left\lfloor\frac{n}{2}\right\rfloor+1$.

Proof. Combining Proposition 2.1 and Lemma 2.2, we have $I^{\prime}\left(P_{n}\right)=\left\lfloor\frac{n}{2}\right\rfloor+1$ or $\left\lfloor\frac{n}{2}\right\rfloor$. Suppose $I^{\prime}\left(P_{n}\right)=\left\lfloor\frac{n}{2}\right\rfloor$. Then there exists a code $W^{\prime}$ of size $\left\lfloor\frac{n}{2}\right\rfloor$ such that $W^{\prime}$ separates any pair of distinct vertices of $P_{n}$ and $B_{P_{n}}(i) \cap W^{\prime}=\emptyset$ for a unique vertex $i$.

Case 1. $i \neq 0$ and $i \neq n-1$. Then $i-1, i, i+1 \notin W^{\prime}$, and $i-2, i-3, i-4, i+2, i+3, i+4 \in$ $W^{\prime}$, so $4 \leqslant i \leqslant n-5$. If we delete the six vertices $i-1, i, i+1, i+2, i+3, i+4$, and connect $i-2$ by an edge to $i+5$, then we get an identifying code of $P_{n-6}$. Hence $\left|W^{\prime}\right| \geqslant 3+I\left(P_{n-6}\right)=\left\lfloor\frac{n}{2}\right\rfloor+1$, a contradiction.

Case 2. $i=0$ or $n-1$. Without loss of generality, assume that $i=n-1$. Then $n-1, n-2 \notin W^{\prime}$, and $n-3, n-4, n-5 \in W^{\prime}$. If $\{0,1, \ldots, n-6\} \subseteq W^{\prime}$, then $\left|W^{\prime}\right|=n-2 \geqslant$ $\left\lfloor\frac{n}{2}\right\rfloor+1$, a contradiction. Now suppose $\{0,1, \ldots, n-6\} \nsubseteq W^{\prime}$. Take the smallest $k \geqslant 6$ such that $n-k \notin W^{\prime}$. If $k=n-1$ or $k=n$, then $\left|W^{\prime}\right| \geqslant n-3 \geqslant\left\lfloor\frac{n}{2}\right\rfloor+1$, a contradiction. It is clear that $k \neq n-2$. For $k \leqslant n-3$, by deleting the vertices $n-k, n-k+1, \ldots, n-1$, we get an identifying code of $P_{n-k}$. It follows that $\left|W^{\prime}\right| \geqslant k-3+I\left(P_{n-k}\right) \geqslant\left\lfloor\frac{n}{2}\right\rfloor+1$, a contradiction.

Therefore, $I^{\prime}\left(P_{n}\right)=\left\lfloor\frac{n}{2}\right\rfloor+1$. Since $I^{\prime \prime}\left(P_{n}\right)=I^{\prime}\left(P_{n}\right)$ for $I^{\prime}\left(P_{n}\right) \geqslant 4$, the desired result follows.

Proposition 2.4 $I^{\prime}\left(C_{n}\right)=I^{\prime \prime}\left(C_{n}\right)= \begin{cases}\frac{n}{2}, & n \text { is even and } n \geqslant 8 \\ \frac{n+3}{2}, & n \text { is odd and } n \geqslant 13 .\end{cases}$ 
Proof. Note that $I^{\prime \prime}\left(C_{n}\right)=I^{\prime}\left(C_{n}\right)$ for $I^{\prime}\left(C_{n}\right) \geqslant 4$. Combining Proposition 2.1 and Lemma 2.2 , we only need to prove $I^{\prime}\left(C_{n}\right) \geqslant I\left(C_{n}\right)$. It is routine to show that $I^{\prime}\left(C_{n}\right) \geqslant$ $I\left(C_{n}\right)$ for $n=8$ or $n=10$. Next, we consider $n \geqslant 12$. Let $W^{\prime}$ be a code of size $I^{\prime}\left(C_{n}\right)$ such that $W^{\prime}$ separates any pair of distinct vertices of $C_{n}$. If $W^{\prime}$ is an identifying code, then $I^{\prime}\left(C_{n}\right)=\left|W^{\prime}\right| \geqslant I\left(C_{n}\right)$. Now suppose that $W^{\prime}$ is not an identifying code. Then there exists a unique vertex $i \in V\left(C_{n}\right)$ such that $\{i-1, i, i+1\} \cap W^{\prime}=\emptyset$, which implies that $\{i-2, i-3, i-4, i+2, i+3, i+4\} \subseteq W^{\prime}$. If we delete the six vertices $i-1, i, i+1, i+2, i+3, i+4$, and connect $i-2$ by an edge to $i+5$, then we get an identifying code of $C_{n-6}$. Therefore $I^{\prime}\left(C_{n}\right)=\left|W^{\prime}\right| \geqslant 3+I\left(C_{n-6}\right)=I\left(C_{n}\right)$, as desired.

\section{Main results}

We always assume that $G$ is a connected graph and $H$ is an arbitrary graph. In this section, we first provide the sufficient and necessary condition for $G[H]$ to be identifiable. Moreover, if $G[H]$ is identifiable, we determine the minimum cardinality of identifying codes of $G[H]$ in terms of the order of $G$ and the two parameters of $H$ given in Section 2 .

The lexicographic product $G[H]$ of graphs $G$ and $H$ is the graph with the vertex set $\{(u, v) \mid u \in V(G), v \in V(H)\}$, and the edge set $\left\{\left\{\left(u_{1}, v_{1}\right),\left(u_{2}, v_{2}\right)\right\} \mid d_{G}\left(u_{1}, u_{2}\right)=1\right.$, or $u_{1}=$ $u_{2}$ and $\left.d_{H}\left(v_{1}, v_{2}\right)=1\right\}$. For any two distinct vertices $\left(u_{1}, v_{1}\right),\left(u_{2}, v_{2}\right)$ of $G[H]$, we observe that

$$
d_{G[H]}\left(\left(u_{1}, v_{1}\right),\left(u_{2}, v_{2}\right)\right)= \begin{cases}1, & \text { if } u_{1}=u_{2}, d_{H}\left(v_{1}, v_{2}\right)=1 \\ 2, & \text { if } u_{1}=u_{2}, d_{H}\left(v_{1}, v_{2}\right) \geqslant 2 \\ d_{G}\left(u_{1}, u_{2}\right), & \text { if } u_{1} \neq u_{2}\end{cases}
$$

For $u \in V(G)$, let $N_{G}(u)=B_{G}(u) \backslash\{u\}$. For any $u_{1}, u_{2} \in V(G)$, define $u_{1} \equiv u_{2}$ if and only if $B_{G}\left(u_{1}\right)=B_{G}\left(u_{2}\right)$ or $N_{G}\left(u_{1}\right)=N_{G}\left(u_{2}\right)$. Hernando et al. [9] proved that " $\equiv$ " is an equivalent relation and the equivalence class of a vertex is of three types: a class of size 1 , a clique of size at least 2 , an independent set of size at least 2 . Denote all equivalence classes by

$$
W_{1}, \ldots, W_{p}, U_{1}, \ldots, U_{k}, V_{1}, \ldots, V_{l}
$$

where

(i) $\left|W_{q}\right|=1, q=1, \ldots, p$;

(ii) for any $u_{1}, u_{2} \in U_{i}, i=1, \ldots, k, B_{G}\left(u_{1}\right)=B_{G}\left(u_{2}\right)$;

(iii) for any $u_{1}, u_{2} \in V_{j}, j=1, \ldots, l, N_{G}\left(u_{1}\right)=N_{G}\left(u_{2}\right)$.

Denote $s(G)=\left|U_{1}\right|+\cdots+\left|U_{k}\right|-k, t(G)=\left|V_{1}\right|+\cdots+\left|V_{l}\right|-l$.

For $u \in V(G)$ and $C \subseteq V(H)$, let $C^{u}=\{(u, v) \mid(u, v) \in V(G[H]), v \in C\}$. For $S \subseteq V(G[H])$, let $S_{u}=\{v \mid v \in V(H),(u, v) \in S\}$. Note that $\left(S_{u}\right)^{u}=H^{u} \cap S$, where $H^{u}=(V(H))^{u}$. By $(1)$, we have

$$
\begin{gathered}
B_{G[H]}((u, v))=\left(B_{H}(v)\right)^{u} \cup \bigcup_{w \in N_{G}(u)} H^{w}, \\
B_{G[H]}((u, v)) \cap S=\left(\left(B_{H}(v)\right) \cap S_{u}\right)^{u} \cup \bigcup_{w \in N_{G}(u)}\left(S_{w}\right)^{w} .
\end{gathered}
$$


Theorem 3.1 Let $G$ be a connected graph and $H$ be an arbitrary graph. Then the lexicographic product $G[H]$ of $G$ and $H$ is identifiable if and only if

(i) $H$ is identifiable and $\Delta(H) \leqslant|V(H)|-2$; or

(ii) both $G$ and $H$ are identifiable.

Proof. Suppose $G[H]$ is identifiable. If $H$ is not identifiable, then there exist two distinct vertices $v_{1}, v_{2}$ of $H$ with $B_{H}\left(v_{1}\right)=B_{H}\left(v_{2}\right)$. By $(3), B_{G[H]}\left(\left(u, v_{1}\right)\right)=B_{G[H]}\left(\left(u, v_{2}\right)\right)$ for $u \in V(G)$. This contradicts the condition that $G[H]$ is identifiable.

If $\Delta(H)=|V(H)|-1$ and $G$ is not identifiable, then there exist $v \in V(H)$ and two distinct vertices $u_{1}, u_{2}$ of $G$ such that

$$
B_{H}(v)=V(H) \text { and } B_{G}\left(u_{1}\right)=B_{G}\left(u_{2}\right) .
$$

By (3), we have

$$
B_{G[H]}\left(\left(u_{1}, v\right)\right)=H^{u_{1}} \cup \bigcup_{u \in N_{G}\left(u_{1}\right)} H^{u}=\bigcup_{u \in B_{G}\left(u_{1}\right)} H^{u}=\bigcup_{u \in B_{G}\left(u_{2}\right)} H^{u}=B_{G[H]}\left(\left(u_{2}, v\right)\right),
$$

which contradicts the condition that $G[H]$ is identifiable.

Therefore, (i) or (ii) holds.

Conversely, suppose (i) or (ii) holds. Assume that $G[H]$ is not identifiable. Therefore, there exist two distinct vertices $\left(u_{1}, v_{1}\right)$ and $\left(u_{2}, v_{2}\right)$ such that $B_{G[H]}\left(\left(u_{1}, v_{1}\right)\right)=$ $B_{G[H]}\left(\left(u_{2}, v_{2}\right)\right)$. If $u_{1} \neq u_{2}$, then $d_{G}\left(u_{1}, u_{2}\right)=1$. It follows that $B_{G}\left(u_{1}\right)=B_{G}\left(u_{2}\right)$ and $B_{H}\left(v_{1}\right)=B_{H}\left(v_{2}\right)=V(H)$, contrary to (i) and (ii). If $u_{1}=u_{2}$, by (3), one gets $B_{H}\left(v_{1}\right)=B_{H}\left(v_{2}\right)$, contrary to the condition that $H$ is identifiable.

Remark 3.1 Let $r$ be a positive integer and $\Gamma$ be a graph. Given a vertex $v \in V(\Gamma)$, define $B_{\Gamma}^{(r)}(v)=\left\{u \mid u \in V(\Gamma), d_{\Gamma}(u, v) \leqslant r\right\}$. An $r$-identifying code of $\Gamma$ is a code which $r$-covers all the vertices of $\Gamma$ and $r$-separates any pair of distinct vertices of $\Gamma$ (see [12] for details). Identifying codes in this paper are 1-identifying codes. If $r \geqslant 2$, then $G[H]$ does not admit any $r$-identifying code. Indeed, by $(1), B_{G[H]}^{(r)}\left(\left(u, v_{1}\right)\right)=B_{G[H]}^{(r)}\left(\left(u, v_{2}\right)\right)$ for $r \geqslant 2$.

Lemma 3.2 Let $G$ be a connected graph and $H$ be an arbitrary graph. If $S$ is an identifying code of $G[H]$, then for any vertex $u$ of $G, S_{u}$ separates any pair of distinct vertices of $H$. Moreover, with reference to (2), the following hold.

(i) If $k \neq 0$, then there exists at most one vertex $u \in U_{i}$ satisfying $S_{u} \subseteq B_{H}(v)$ for a vertex $v$ of $H$, where $i=1, \ldots, k$;

(ii) If $l \neq 0$, then there exists at most one vertex $u \in V_{j}$ satisfying $S_{u} \cap B_{H}(v)=\emptyset$ for a vertex $v$ of $H$, where $j=1, \ldots, l$.

Proof. Assume that there exist $u_{0} \in V(G)$ and two distinct vertices $v_{1}, v_{2}$ of $H$ such that $S_{u_{0}} \cap B_{H}\left(v_{1}\right)=S_{u_{0}} \cap B_{H}\left(v_{2}\right)$. By (4), $B_{G[H]}\left(\left(u_{0}, v_{1}\right)\right) \cap S=B_{G[H]}\left(\left(u_{0}, v_{2}\right)\right) \cap S$, contrary to the condition that $S$ is an identifying code of $G[H]$. 
(i) Assume that there exist two distinct vertices $u_{1}, u_{2} \in U_{i}$ such that $S_{u_{1}} \subseteq B_{H}\left(v_{1}\right)$ and $S_{u_{2}} \subseteq B_{H}\left(v_{2}\right)$. Since $B_{G}\left(u_{1}\right)=B_{G}\left(u_{2}\right)$, by (4) we have

$$
B_{G[H]}\left(\left(u_{1}, v_{1}\right)\right) \cap S=\left(S_{u_{1}}\right)^{u_{1}} \cup \bigcup_{u \in N_{G}\left(u_{1}\right)}\left(S_{u}\right)^{u}=\bigcup_{u \in B_{G}\left(u_{2}\right)}\left(S_{u}\right)^{u}=B_{G[H]}\left(\left(u_{2}, v_{2}\right)\right) \cap S .
$$

Since $S$ is an identifying code of $G[H]$, we have $\left(u_{1}, v_{1}\right)=\left(u_{2}, v_{2}\right)$, a contradiction.

(ii) Assume that there exist two different vertices $u_{1}, u_{2} \in V_{j}$ such that $S_{u_{1}} \cap B_{H}\left(v_{1}\right)=$ $S_{u_{2}} \cap B_{H}\left(v_{2}\right)=\emptyset$. Since $N_{G}\left(u_{1}\right)=N_{G}\left(u_{2}\right)$, by (4) we have

$$
B_{G[H]}\left(\left(u_{1}, v_{1}\right)\right) \cap S=\bigcup_{u \in N_{G}\left(u_{1}\right)}\left(S_{u}\right)^{u}=\bigcup_{u \in N_{G}\left(u_{2}\right)}\left(S_{u}\right)^{u}=B_{G[H]}\left(\left(u_{2}, v_{2}\right)\right) \cap S .
$$

Since $S$ is an identifying code of $G[H]$, we obtain $\left(u_{1}, v_{1}\right)=\left(u_{2}, v_{2}\right)$, a contradiction.

In equivalence classes $(2)$ of $V(G)$, choose $\bar{u}_{i} \in U_{i}, i=1, \ldots, k$, and $\bar{v}_{j} \in V_{j}, j=$ $1, \ldots, l$. Let $\bar{W}_{0}=\cup_{q=1}^{p} W_{q} \cup\left\{\bar{u}_{1}, \ldots, \bar{u}_{k}, \bar{v}_{1}, \ldots, \bar{v}_{l}\right\}$ and $\bar{U}_{i}=U_{i} \backslash\left\{\bar{u}_{i}\right\}, i=1, \ldots, k$, $\bar{V}_{j}=V_{j} \backslash\left\{\bar{v}_{j}\right\}, j=1, \ldots, l$. Therefore, we have a partition of $V(G)$ :

$$
\bar{W}_{0}, \bar{U}_{1}, \ldots, \bar{U}_{k}, \bar{V}_{1}, \ldots, \bar{V}_{l} .
$$

Lemma 3.3 Let $C$ be an identifying code of graph $H$, and let $C^{\prime}, C^{\prime \prime}$ be two codes which separate any pair of distinct vertices of $H$ and $C^{\prime \prime} \nsubseteq B_{H}(v)$ for every vertex $v$ of $H$. With reference to (5),

$$
S=\bigcup_{u \in \bar{W}_{0}}\left(C^{\prime}\right)^{u} \cup \bigcup_{i=1}^{k} \bigcup_{u \in \bar{U}_{i}}\left(C^{\prime \prime}\right)^{u} \cup \bigcup_{i=1}^{l} \bigcup_{u \in \bar{V}_{i}} C^{u}
$$

is an identifying code of $G[H]$.

Proof. For any $u \in V(G)$, we have

$$
S_{u}= \begin{cases}C^{\prime}, & \text { if } u \in \bar{W}_{0}, \\ C^{\prime \prime}, & \text { if } u \in \cup_{i=1}^{k} \bar{U}_{i}, \\ C, & \text { if } u \in \cup_{j=1}^{l} \bar{V}_{j} .\end{cases}
$$

Since $G$ is connected, there exists a vertex $w$ adjacent to $u$. By (1), $S$ covers all vertices of $G[H]$. Hence, we only need to show that, for any two distinct vertices $\left(u_{1}, v_{1}\right)$ and $\left(u_{2}, v_{2}\right)$ of $G[H]$

$$
B_{G[H]}\left(\left(u_{1}, v_{1}\right)\right) \cap S \neq B_{G[H]}\left(\left(u_{2}, v_{2}\right)\right) \cap S .
$$

If $u_{1}=u_{2}$, the fact that $S_{u_{1}}$ separates $v_{1}$ and $v_{2}$ implies $B_{H}\left(v_{1}\right) \cap S_{u_{1}} \neq B_{H}\left(v_{2}\right) \cap S_{u_{1}}=$ $B_{H}\left(v_{2}\right) \cap S_{u_{2}}$, so (6) holds by (4). Now suppose that $u_{1} \neq u_{2}$. In order to prove (6), it is sufficient to show that there exists $\left(u_{0}, v_{0}\right) \in S$ such that

$$
d_{G[H]}\left(\left(u_{0}, v_{0}\right),\left(u_{1}, v_{1}\right)\right) \leqslant 1, d_{G[H]}\left(\left(u_{0}, v_{0}\right),\left(u_{2}, v_{2}\right)\right) \geqslant 2
$$


or

$$
d_{G[H]}\left(\left(u_{0}, v_{0}\right),\left(u_{2}, v_{2}\right)\right) \leqslant 1, d_{G[H]}\left(\left(u_{0}, v_{0}\right),\left(u_{1}, v_{1}\right)\right) \geqslant 2 .
$$

Case 1. $u_{1} \not \equiv u_{2}$. Then there exists $u_{0} \in V(G) \backslash\left\{u_{1}, u_{2}\right\}$ such that $d_{G}\left(u_{1}, u_{0}\right)=1$ and $d_{G}\left(u_{2}, u_{0}\right) \geqslant 2$, or $d_{G}\left(u_{1}, u_{0}\right) \geqslant 2$ and $d_{G}\left(u_{2}, u_{0}\right)=1$. Take $v_{0} \in S_{u_{0}}$. Then $\left(u_{0}, v_{0}\right) \in S$. By (1), (7) or (8) holds.

Case 2. $B_{G}\left(u_{1}\right)=B_{G}\left(u_{2}\right)$. Then $u_{1}$ and $u_{2}$ are adjacent and fall into some $U_{i}$. It follows that $u_{1} \in \bar{U}_{i}$ or $u_{2} \in \bar{U}_{i}$. Without loss of generality, suppose $u_{1} \in \bar{U}_{i}$. Pick $u_{0}=u_{1}$. Since $C^{\prime \prime} \nsubseteq B_{H}\left(v_{1}\right)$, there exists $v_{0} \in C^{\prime \prime}$ such that $\left(u_{0}, v_{0}\right) \in S$ and $d_{H}\left(v_{0}, v_{1}\right) \geqslant 2$. Then (8) holds by (1).

Case 3. $N_{G}\left(u_{1}\right)=N_{G}\left(u_{2}\right)$. Then $u_{1}$ and $u_{2}$ are at distance 2 and fall into some $V_{j}$. It follows that $u_{1} \in \bar{V}_{j}$ or $u_{2} \in \bar{V}_{j}$. Without loss of generality, suppose $u_{1} \in \bar{V}_{j}$. Pick $u_{0}=u_{1}$. Since $C$ covers $v_{1}$, there exists $v_{0} \in C$ such that $\left(u_{0}, v_{0}\right) \in S$ and $d_{H}\left(v_{0}, v_{1}\right) \leqslant 1$. By (1), (7) holds.

Theorem 3.4 Let $G$ be a connected graph and $H$ be an arbitrary graph. Suppose (i) or (ii) holds in Theorem 3.1.

(i) If $\Delta(H) \leqslant|V(H)|-2$, then

$$
I(G[H])=(|V(G)|-s(G)-t(G)) I^{\prime}(H)+s(G) I^{\prime \prime}(H)+t(G) I(H) ;
$$

(ii) If $\Delta(H)=|V(H)|-1$, then

$$
I(G[H])=(|V(G)|-t(G)) I^{\prime}(H)+t(G) I(H) .
$$

Proof. (i) By Theorem 3.1, $I(H)$ and $I^{\prime}(H)$ are well defined. Since $V(H)$ separates any pair of distinct vertices of $H$ and $V(H) \nsubseteq B_{H}(v)$ for every $v \in V(H), I^{\prime \prime}(H)$ is well defined. Let $S$ be an identifying code of $G[H]$ with the minimum cardinality, by Lemma 3.2 ,

$$
\begin{aligned}
I(G[H]) & =|S|=\sum_{i=1}^{p} \sum_{u \in W_{i}}\left|S_{u}\right|+\sum_{i=1}^{k} \sum_{u \in U_{i}}\left|S_{u}\right|+\sum_{i=1}^{l} \sum_{u \in V_{i}}\left|S_{u}\right| \\
& \geqslant(p+k+l) I^{\prime}(H)+\left(\sum_{i=1}^{k}\left|U_{i}\right|-k\right) I^{\prime \prime}(H)+\left(\sum_{i=1}^{l}\left|V_{i}\right|-l\right) I(H) \\
& =(|V(G)|-s(G)-t(G)) I^{\prime}(H)+s(G) I^{\prime \prime}(H)+t(G) I(H) .
\end{aligned}
$$

By Lemma 3.3 we can construct an identifying code of $G[H]$ with cardinality $(|V(G)|-$ $s(G)-t(G)) I^{\prime}(H)+s(G) I^{\prime \prime}(H)+t(G) I(H)$. Therefore, (9) holds.

(ii) By Theorem 3.1, both $G$ and $H$ are identifiable. So $I(H)$ and $I^{\prime}(H)$ are well defined. Owing to $B_{G}\left(u_{1}\right) \neq B_{G}\left(u_{2}\right)$ for any two distinct vertices $u_{1}, u_{2}$ of $G$, we get $k=0$ in (2) and (5). Similar to the proof of (i), (10) holds.

Combining Propositions 2.1, 2.3, 2.4 and Theorem 3.4, we have the following results.

Corollary 3.5 Let $G$ be a connected graph of order $m(m \geqslant 2)$.

(i) For $n \geqslant 7, I\left(G\left[P_{n}\right]\right)=m\left(\left\lfloor\frac{n}{2}\right\rfloor+1\right)$;

(ii) For $n \geqslant 12, I\left(G\left[C_{n}\right]\right)= \begin{cases}\frac{m n}{2}, & n \text { is even, } \\ \frac{m(n+3)}{2}, & n \text { is odd. }\end{cases}$ 
Acknowledgement: The authors are indebted to the anonymous reviewer for providing simple proofs of Propositions 2.3 and 2.4. This research is supported by NSFC(11271047), SRFDP and the Fundamental Research Funds for the Central University of China.

\section{References}

[1] Y. Ben-Haim, S. Litsyn, Exact minimum density of codes identifying vertices in the square grid, SIAM J. Discrete Math. 19 (2005) 69-82.

[2] N. Bertrand, I. Charon, O. Hudry, A. Lobstein, Identifying and locating-dominating codes on chains and cycles, European J. Combin. 25 (2004) 969-987.

[3] U. Blass, I. Honkala, S. Litsyn, On binary codes for identification, J. Combin. Designs 8 (2000) $151-156$.

[4] I. Charon, O. Hudry, A. Lobstein, Minimizing the cardinality of an identifying or locating-dominating code in a graph is NP-hard, Theoret. Comp. Sci. 290 (2003) 2109-2120.

[5] G. Cohen, I. Honkala, A. Lobstein, G. Zémor, On identifying codes, in: A. Barg, S. Litsyn(Eds.), Codes and Association Schemes, in: DIMACS Series, vol.56, American Mathematical Society, Providence, RI (2001) 97-109.

[6] G. Cohen, S. Gravier, I. Honkala, A. Lobstein, M. Mollard, C. Payan, G. Zémor, Improved identifying codes for the grid, Electron. J. Combin. 6 (1999) R19 Comments.

[7] S. Gravier, J. Moncel, A. Semri, Identifying codes of cycles, European J. Combin. 27 (2006) 767-776.

[8] S. Gravier, J. Moncel, A. Semri, Identifying codes of Cartesian product of two cliques of the same size, Electron. J. Combin. 15 (2008) N4.

[9] C. Hernando, M. Mora, I. M. Pelayo, C. Seara and D. R. Wood, Extremal graph theory for metric dimension and diameter, Electronic J. Combin. 17 (2010) R30.

[10] I. Honkala, T. Laihonen, On identifying codes in the triangular and square grids, SIAM J. Comput. 33 (2004) 304-312.

[11] I. Honkala, A. Lobstein, On identifying codes in binary Hamming spaces, J. Combin. Theory Ser. A 99 (2002) 232-243.

[12] M.G. Karpovsky, K. Chakrabarty, L.B. Levitin, On a new class of codes for identifying vertices in graphs, IEEE Trans. Inform. Theory 44 (1998) 599-611.

[13] M.G. Karpovsky, K. Chakrabarty, L.B. Levitin, D.R. Avresky, On the covering of vertices for fault diagnosis in hypercubes, Inform. Process. Lett. 69 (1999) 99-103.

[14] J. Moncel, Monotonicity of the minimum cardinality of an identifying code in the hypercube, Discrete Appl. Math. 154 (2006) 898-899.

[15] D.F. Rall, K. Wash, Identifying codes of the direct product of two cliques, arXiv:1206.3596v1 [math. CO], 2012.

[16] M. Xu, K. Thulasiraman, X. Hu, Identifying codes of cycles with odd orders, European J. Combin. 29 (2008) 1717-1720. 